Nig. J. Biotech. Vol. 38 (2) : 73-83 (Dec 2021)

ISSN: 01891731

Available online at

http://www.ajol.info/index.php/njb/index

and www.biotechsocietynigeria.org

DOI: https://dx.doi.org/10.4314/njb.v38i2.8

\title{
Potential of Internet of Things and Semantic Web Technologies in the Health Sector
}

\author{
${ }^{*}$ Edeh, M. 0. ${ }^{1}$, Otto, E. E. ${ }^{2}$, Richard-Nnabu, N. E. ${ }^{3}$, Ugboaja, S. G. ${ }^{4}$, \\ Umoke, C. C. ${ }^{5}$, and Omachi, $D^{6}$. \\ ${ }^{1}$ Department of Mathematics and Computer Science, Coal City University, Nigeria \\ ${ }^{2}$ Department of Environmental Health Science, POGIL College of Health Technology, \\ Ijebu Ode, Ogun State, Nigeria \\ ${ }^{3}$ Department of Computer Science/Informatics, Alex Ekwueme Federal University Ndufu \\ Alike Ikwo, (AE-FUNAI), Abakaliki, Nigeria \\ ${ }^{4}$ Department of Computer Science, Michael Okpara University of Agriculture, Nigeria \\ ${ }^{5}$ Dept. of Vocational and technical Education, Alex Ekwueme Federal University Ndufu Alike \\ Ikwo,(AE-FUNAI), Abakaliki, Nigeria \\ ${ }^{6}$ Department of Educational Foundation, Peaceland College of Education, Enugu, Nigeria
}

\begin{abstract}
Science and technology have become essential components of healthcare. The emergence of Artificial intelligence (AI), Blockhain, mobile techs, Internet of Things (IoT) and Semantic Web Technologies (SWTs) etcetera have amplified the growing influence of technology in global health systems. IoT and SWTs in particular are seen as the next-generation technologies that can revolutionize global health systems. Thus, this paper examines the various potentials of IOT and SWTs in the health sector, with a view to creating more awareness regarding its prospects and associated risks, and also to assist stakeholders to maximize its possibilities to enrich healthcare delivery. We deduced that both IoT and SWTs harbour promising features that are now being deployed in the health sector to enhance quality service delivery, medical research and innovations, smart health, telemedicine, communications, patient care and effective health outcomes just to mention but a few. We conclude that the adoption and implementation of IoT-based devices and SWTs will be significant in sustainability and improvement of healthcare systems in the future. However, privacy and medical data security remains a major source of concern to many stakeholders.
\end{abstract}

Keywords: Internet of Things, Semantic web technology, e-health, Telemedicine, Ontology, Healthcare

*Corresponding author e-mail: michael.edeh@ccu.edu.ng, Tel: +2347039009048

\section{Introduction}

The internet revolution has brought about tremendous changes to every aspect of human lives, particularly the health sector. The use of the internet and many other emerging technologies such as IoT, SWTs, Artificial intelligence (AI), nanotechnology, blockchain, machine learning and mobile technologies are now driving medical innovations that are being adopted globally to improve healthcare. Diagnostic imaging, Electronic Medical Record (EMR) and clinical decision support systems help doctors to diagnose, treat and track patients through their journey to recovery (Mahadevaiah, 2020). Onyema et al (2020a) opined that technology such as Internet of Things (IoT) presents more opportunities for medical educators and 
researchers to improve their job performances.

The Internet of Things is an emerging technology that encompasses many networked devices that can easily communicate and share resources with each other. IoT provides enhancement and better solutions in the medical field, like proper medical record-keeping, sampling, integration of devices, and causes of diseases (Mohd and Ibrahim, 2021). IoT represents a network of billions of connecting devices with intelligence and flexibility to communicate and exchange data that can then be analyzed to unlock a wealth of intelligence useful for planning, management and decision making in the health sector (Patel and Patel, 2016). IoT is used for health analytics by physicians and hospitals for years in different areas, including temperature and heart rate monitoring, data protection, and communication. Some of the important services offered by IoT in healthcare include: telemedicine, contact tracing, robotic sanitisation, diagnosis emergency alert, data privacy and storage. Both Non-wearable IoT devices as ingestible sensors and wearable IoT devices like smart watches can provide necessary information about the patient's heart rate, blood sugar level, blood oxygen level, and so on thereby minimizing human interference or assistance. With IoT and Semantic web technologies, health institutions or physicians can now interact better with patients, keep track of their health continuously and make prescriptions or decisions accordingly. Umair t al (2021) stated that users find it hard to adopt IoT systems for healthcare due to energy requirements, lack of skills, security and privacy issues.

Meanwhile, semantic web technologies define an enrichment of the syntactic web where information is contained with its meaning. Semantic Web (SW) consists of documents as well as data on the World Wide Web that can be assembled, executed, and transformed by machines more efficiently (Tiwari et al, 2019). The Semantic Web is very similar to the World Wide Web (WWW), though it appears to be an advancement that gives the Web far greater utility. Semantic web presents many opportunities in healthcare, particularly as it relates to automated medical decision support system; rule-based healthcare system, Interoperability and use of ontologies in healthcare delivery. Semantic web enhances the quality of health information available to patients giving them the ability to choose from a list of quality health providers.

The objective of this paper is to examine the potentials of IoT and Semantic Web technologies in transforming services and outcomes in the health sector. The paper is organized into the various sections under the following headings as follows: Introduction, Review of related work, Application of IoT in healthcare, Limitations of IoT in healthcare, Application of Semantic web technologies in healthcare and then closely followed by conclusion and future perspectives to overcome the various limitations confronting the adoption of IoT and SWTs in the health sector.

\section{Review of Related Work}

Emerging technologies are being applied in different aspects in health settings. For instance, technology proved very useful in medical research, treatment, diagnosis education and tracking of patients during the recent wave of COVID-19 pandemic (Onyema et al, 2020b). According to Islam et al (2015) the aim of the application of IoT healthcare services is to provide an improved user experience at low cost and enhanced access. Wearable IoT technology devices like smart watches and real-time position systems such as Radio Frequency Identification (RFID) bracelets were very useful in personalized health services, contact tracing and monitoring of patients under different conditions as seen in figure1. 


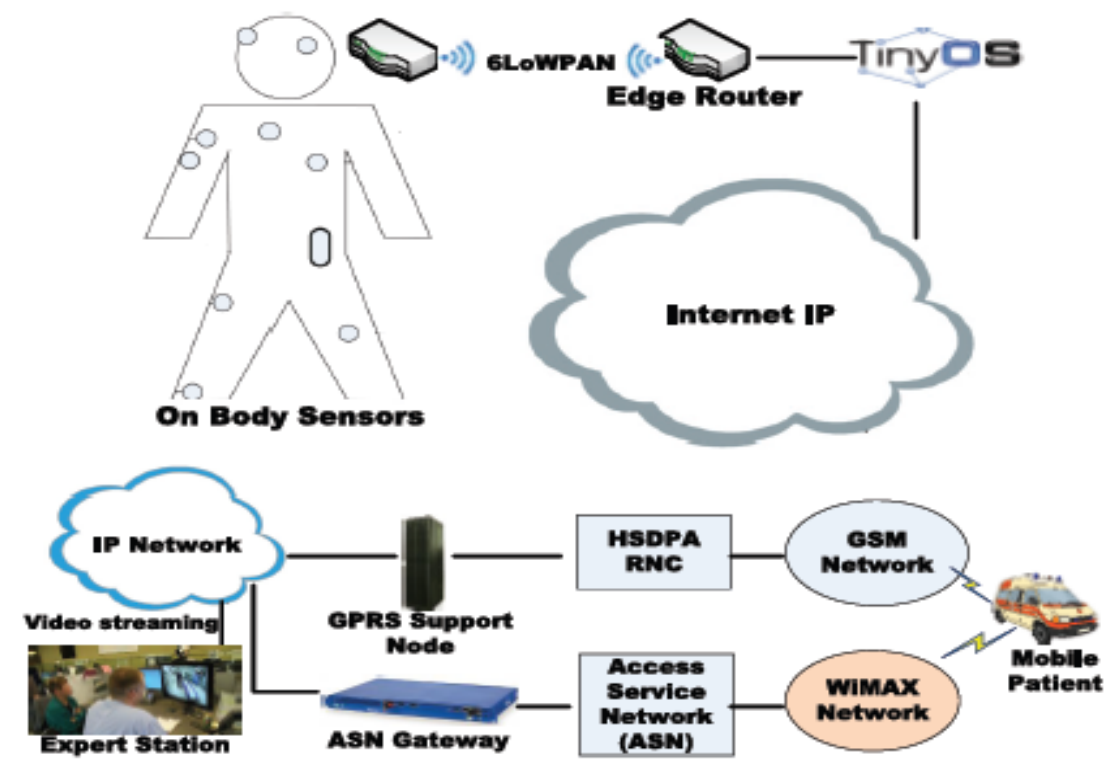

Figure 1: IoT-enhanced Remote Monitoring in wearables and personalized healthcare (Islam et al, 2015).

David and Kyle (2015) suggested that IoT-enabled healthcare would provide digitalized patient data to aid e-health (remote patient diagnostics and monitoring, telehealth and more effective treatment that is affordable and accessible). Partha (2014) presented an IoT-based model named Home Health Hub IoT (H3IoT), for tracking health of elderly persons. The model contains connected biosensors, applications, internet, and the hardware platform, which give physicians and relatives of elderly patients to interact with them and also track their health conditions remotely from their homes. Umair et al (2021) examined the impact of COVID-19 pandemic on IoT adoption. They concluded that the huge demand for effective healthcare driven by overwhelmed health system due to COVID-19 has accelerated IoT adoption in the health sector. A study conducted by Yap and Tan (2020) examined the acceptance of IoT-enabled healthcare in Malaysia using Technology Acceptance Model (TAM) model and questionnaire. Their findings showed that $79 \%$ of the respondents accepted IoT-enabled healthcare applications, but many of them were limited by various barriers such as poor infrastructure and regulation issues. Eero et al (2008) provided e-health portal based on shared metadata schema and ontologies. The system has features that enhance updated health information across board and search technique to ease the search for medical data or record using semantic web technologies.

Ali and Samina (2009) designed an ontology-based system for automating clinical pathways. The system describes the integration of multiple localized clinical pathways to unify diseases for prostate cancer management system. Similarly, Verma et al (2017) introduced a coud-centric IoT-based student healthcare monitoring framework that consists of three phases. The Verma et al (2017) model enabled smart wearable body sensors, heart sensors, gastro sensors and Electrocardiogram ECG to monitor and collect health condition data and diagnose patients to determine their heart rate, blood pressure and blood glucose level. The model enabled students to submit answers relating to their health status using their mobiles phones which are later saved in the created cloud repository for action or reference. Shah et al, 2019 carried out a review of the role played by mobile computing in IoT-enabled healthcare. They concluded that mobile computing 
expands the application of IoT in healthcare through the concept of mhealth. Pathak et al (2012) evaluated the use of SWT in Electronic Health Record (EHR) phenotyping by changing EHR data from multiple sources into Resource Description Framework (RDF) graphs. The created RDF graphs were then queried via a single SPARQL endpoint in order to assess research cohort. With the high penetration of mobile technologies and other smart devices (Onyema, 2019), smart health can be made possible by use of smartphones for collection, storage, and exchange of relevant health data among hospitals, health practitioners and patients via connected devices.

\section{Application of Internet of Things (IoT) in Health Sector}

IoT is a generic name for network or connection of physical things that are embedded with sensors, software, and other technologies to facilitate the sharing of resources with other devices and systems over the internet. IoT is about leveraging the power of the internet to link or bring several physical things, people or even computing devices together to achieve seamless communication and exchange of data between people, processes, and things. The emergence of sensor, mobile phones, cloud, machine learning algorithms, Artificial intelligence (AI) and cloud computing technologies have increased the development and influence of IoT devices. The features of Iot and AI have brought about the concept of Smart and interactive health (Onyema et al, 2021). IoT-enabled technologies offer flexibility for system automation, data mining/ analytics and production of smart medical devices and smart diagnosis. The application of IoT in healthcare helps to bring about excellent support systems for physicians and patients especially during emergencies and provision of real-time medical information (Mohd and Ibrahim, 2021). IoT-enabled devices have made remote monitoring in hospitals and other health centres possible, unleashing the potential to keep patients safe and healthy, and empowering health workers to enhance their efficiencies (Wipro website, 2021).

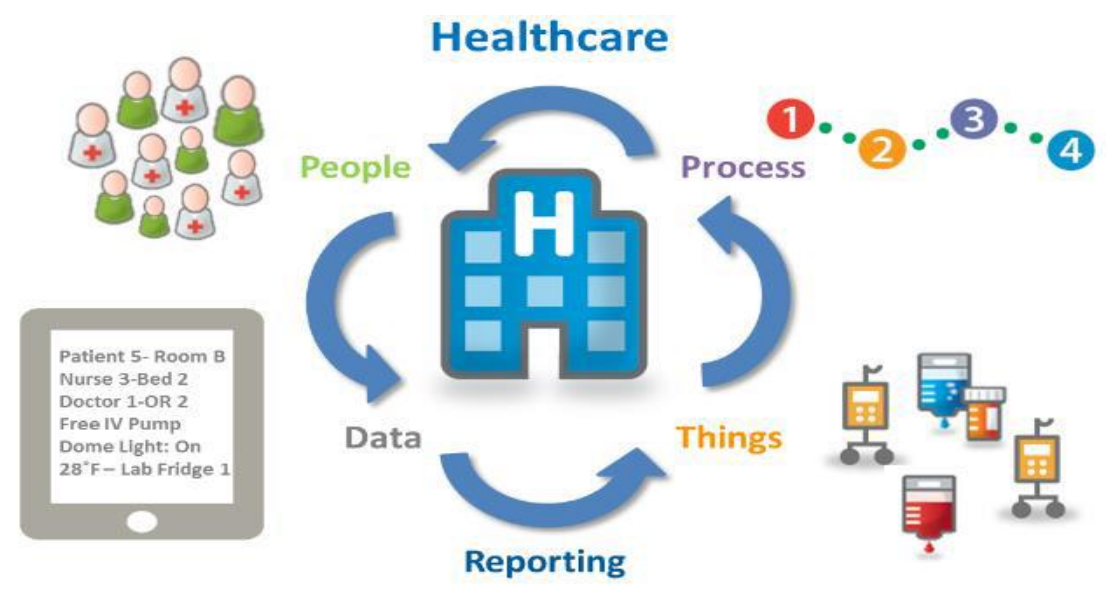

Figure 2: Framework for Application of IoT in Healthcare (Hossain and Muhammad, 2016).

As seen in Figure 2 above, the framework for application of IoT in healthcare is depicted. The Connected objects (Things) sense and collect the health data (Data) of patients (People) and then send it to cloud (private or public cloud). Those data will be analysed (Process) and become a useful health information (Data). Useful health information will be presented (Process) to caregivers (People) in the form of report or monitoring graphic user interface (Yap and Tan, 2020). IoT-based smart medical devices work by tagging technologies like RFID tags, barcodes, QR codes, etc. to obtain data (Hadeel et al, 2021). 
The growing influence of IoT in healthcare brought about the formation of the concept "Internet of Medical Things (IoMT). IoT is changing the space of devices and pace of patients-doctor interactions in providing healthcare solutions leading to automation and mobility health solutions. The application of IoT in the health sector is growing day by day and it has been projected that the market for IoT in healthcare will grow to $\$ 260.75$ billion in 2027 (https://www.reportsanddata.com). Also, research has shown that IoT-related healthcare revenues will climb to more than $\$ 135$ billion in 2025 with 75 billion actively-connected devices across the globe (https://medium.com). IoT technologies can potentially enhance healthcare delivery by connecting patients to health workers, and enabling the collection and analysis of data that can improve patient care. IoT's sensorbased technology provides an excellent capability to reduce the risk of surgery during complicated cases and helpful for COVID-19 type pandemic (Mohd and Ibrahim, 2021). IoT portables assist people to better understand their own health and allow physicians to remotely monitor patients (Shariq et al, 2021). Companies are now taking advantage of IoT to track the health and safety of their employees, which is especially useful for workers employed in risky environments. There is no doubt that effective adoption of IoT would help the global health systems to achieve more robust physician-patient engagement, lower cost and improved health service delivery. According to wipro.com website (2021), the benefits of IoT in the health sector could be summarized as follows:

- Cost Reduction: IoT enables patient monitoring in real time, thus significantly cutting down unnecessary human-human interactions or queues at the hospital.

\section{- Drugs and Equipment} Management: Management of drugs and medical equipment is a major challenge in a healthcare industry. Through connected devices, these are managed and utilized efficiently with reduced costs.

- Error Reduction: Data generated through IoT devices not only help in effective decision making but also ensure smooth healthcare operations with reduced errors, wastes and system costs

- Improved Treatment: It enables physicians to make evidence-based informed decisions and brings absolute transparency and improves treatment outcomes.

\section{- Speedy Disease Diagnosis:}

Continuous patient tracking and real time data helps in diagnosing diseases at an early stage or even before the disease develops based on symptoms. This was applied during the peak of COVID-19 pandemic.

- Proactive Treatment: Continuous health monitoring opens the doors for providing proactive medical diagnosis and more hospital engagements with patients.

- Smart Health: The concept of smart health is being engineered by IoT and Artificial intelligence (AI). With the aid of IoT and AI, many smart health platforms are now being developed to render smart and innovative health services to different categories of patients. Telemedicine are also being enhanced using smart IoT-based mobile devices.

- Dental Health: Some emerging IoT-based technology devices such as Bluetooth-enabled Toothbrush with the help of smartphone apps can record patients' brushing information to study their brushing habits and share the statistics with the dentist (Vermesan and Friess, 2014).

- Patient Monitoring: IoT-based devices enhance the provision of timely data that can be applied in patient surveillance. This helps to 
reduce the burden and frequency of visit to the hospitals. Health workers and physically challenged patients can leverage on this benefit to receive medical care from any location without being physically

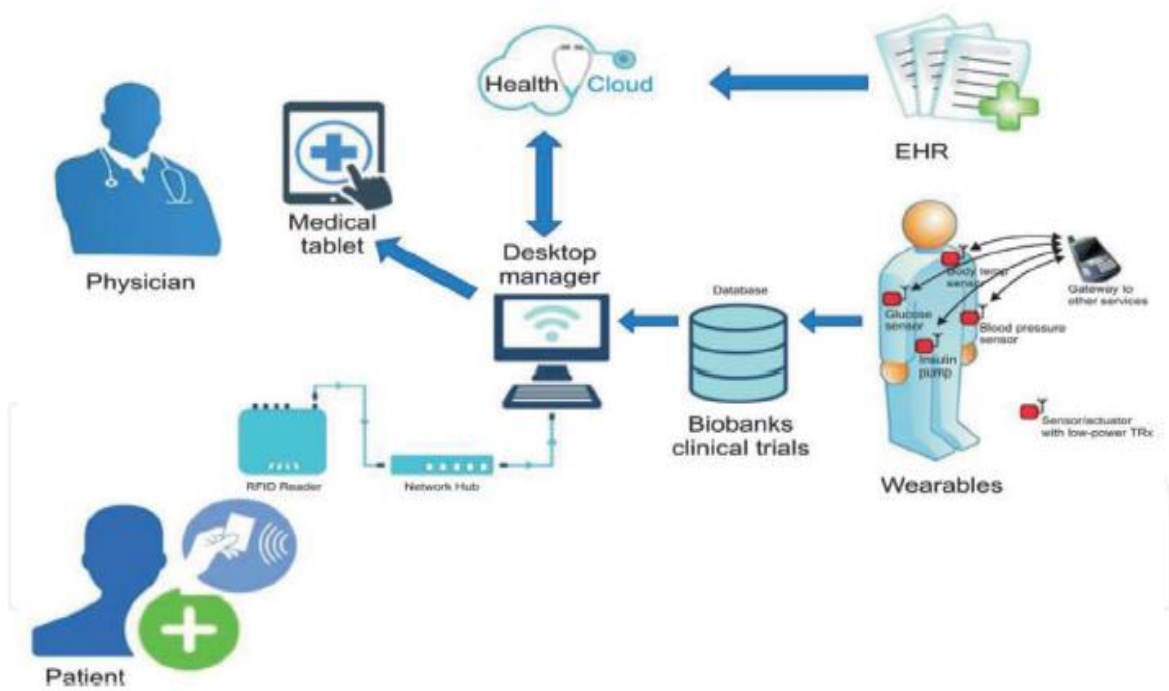

Figure 3: An IoT-powered hospital Scenario ((Dimitrov, 2016)

Figure 3 shows how IoT can be applied in a hospital environment. The patient will have an ID card, which when scanned, links to a secure cloud which stores their electronic health record vitals and lab results and medical and prescription histories (Mohammed and Ahmed, 2019).

\section{Limitations of IoT in Healthcare}

Privacy -Critics of IoT have expressed concerns about the privacy of hospital or patients' sensitive data since the technology often provide or share extreme details of data without the knowledge of the user.

- Complexity -IoT systems and devices often appear very complicated in terms of design, deployment, and maintenance given their use of multiple technologies and a large set of new enabling technologies. Not all healthcare settings or hospitals can afford the resources needed to implement IoT technologies in their systems. This tends to limit its application and deployment.
- Compliance - IoT in healthcare cannot be deployed without complying with medical ethics and regulations. This could pose a challenge to healthcare sector because, its complexity and linkage with other enabling technologies makes it more difficult for users to meet both professional and software compliance standards.

- Security - Security remains a major concern in many technologies and IoT is not an exemption. IoT creates an ecosystem of constantly connected devices communicating over networks. It offers little control despite any security measures. This could expose users or medical data to cyber threats and attacks that may prove very costly.

- Flexibility - Stakeholders in the health sector are concerned about the flexibility of an IoT system to integrate easily with another. Many are perturbed about finding themselves with several conflicting or locked systems. 
- Shortage of Specialist: The adoption of IoT in the health sector demand specialists who are currently in short supply in many health systems and institutions. This limits the ability of hospitals or health practitioners to implement IoT-driven systems and technologies. Unlike advanced countries, many health systems in developing countries still lack the required expertise to integrate IoTrelated devices and enabling technologies.

- Network Issues: IoT is internet based which requires systems or users to connect to reliable networks to enhance its operation and performance. This presents a challenge particularly for rural areas or developing countries where the problem of bad network still persists. Until access to the internet is guaranteed in these areas, the adoption of IoT could prove difficult.

- Power supply issues: The usage of IoT devices in healthcare requires constant power supply to keep the interconnected devices working. This may not be readily available especially in developing countries or rural areas.

\section{Application of Semantic Web Technologies in Healthcare}

Semantic Web Technology helps to annotate data via common ontologies, then interlinks the data on the web and allows effective and easy query of web of knowledge (Erdogan, 2009). This means that SWTs facilitates easy access to medical data on the web. SWT has been gaining a lot of attention in the medical sector in recent time. SWT is designed to provide efficient solutions for the management of complex and distributed data, and to achieve standard interoperability and implementation of web-based knowledge bases (Erdogan, 2009). Semantic Web technologies have the potentials to enhance the storage and sharing of medical knowledge and skills and promote interoperability among healthcare systems (Mahtab and Azin, 2019). Some of the fundamental blocks used in SW are as follows: URI (Universal Resource Identification); XML (Extensible Markup Language); RDF (Resource Description Framework); RDFS (RDF Schema); SPARQL (SPARQL Protocol and RDF Query Language) and OWL (Ontology Web Language). These technologies broaden similar software technologies that underlie the World Wide Web itself.

SWTs are indeed changing medical research and being applied to more general situations like Science Commons, which now aid researchers to openly post data on the internet thereby making their researches more available and accessible to users. Studies have shown that Ontologies provide a conceptual basis for the information exchange; standards create consistency in the information exchange between different systems and allow interoperability between systems. Also, ontologies can facilitate the finding of context-based medical research information so that it can be integrated and used as a foundation for future research and also to create contextbased rules for appointments, procedures, and tests so that the quality of healthcare is improved (Mahtab and Azin, 2019; Hristidis, 2010). However, the main standard for the interoperability in the SWT is the Resource Description Framework (RDF). It is an objectoriented and $\mathrm{XML}$-based standard developed to describe concepts and create documents in semantic web (Mahtab and Azin, 2019).

SWT needs IoT-based solutions for delivering sensor-based services and remote monitoring through association among entities and will provide comfortable and efficient services to the end users (Tiwari et al, 2019). Semantic techniques have demonstrated to be the most pertinent for comprehending and solving a lot of difficulties and challenges which face the healthcare big data community (Rafat et al, 2020). With 
semantic technique, clinical data modelling and integration can be achieved and data can be easily stored, extracted and queried from various sources. For instance, Kang et al. (2014) used HBase repository, ontologies, and data mapping to build a semantic big data model. Similarly, Cheung et al
(2005) implemented a web-based prototype that permitted interoperability between different types of yeast genome data that have different formats. SWT initiates novel method to create and manage data based on semantic metadata which often gives description of data about data.

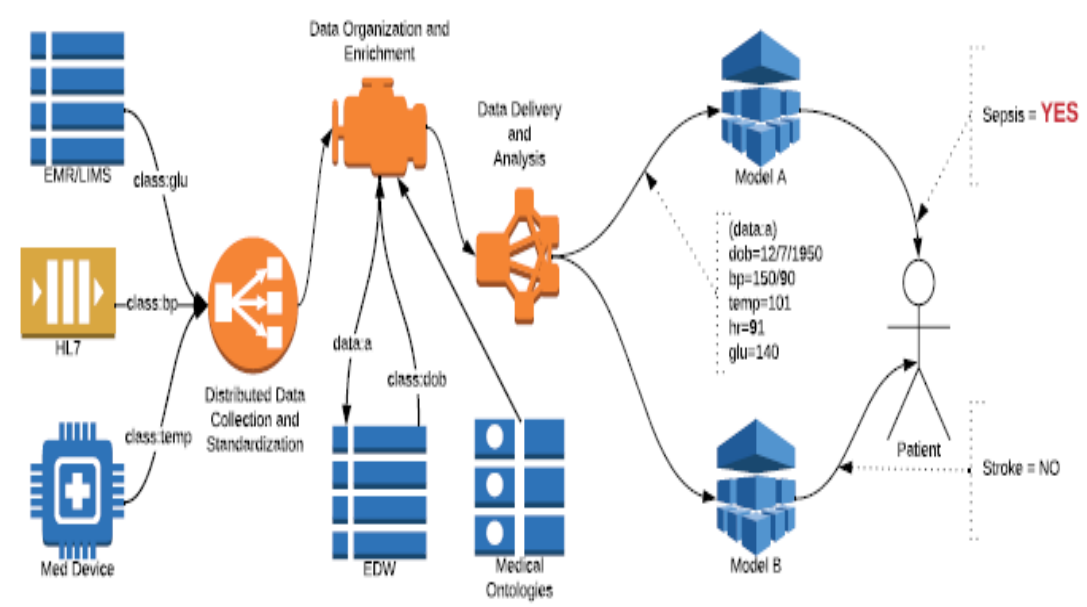

Figure 4: Semantic Engine Use in Clinical Workflow (Daniel and Cody, 2019)

Figure 4 illustrates how a high-level view of how semantic encoder could be used in healthcare workflow to enhance efficiency, including complex event processing (CEP), in which sequence of data are analyzed in search of events in real time using ccomplex event processors, common data models and machine learning algorithms (Daniel and Cody, 2019). Many communities have benefited greatly from the usage of Semantic web technologies in healthcare especially in the areas of big healthcare, smart medical devices and access to customized healthcare (Rafat et al, 2020). Both IoT and SWT provide opportunities for smooth relationship between patients and healthcare providers via seamless and secure connectivity across patients, clinics, and healthcare. They (IoT and SWTs) can both make personalized medicine possible due to their ability to provide smart medical databases and documents on the web. According to Lee et al (2007) "SW functions more behind the scenes than WWW, but its impact can be felt easily. Semantic web will continue to play more critical role to redefine the ability of human and machine to work together in the health sector and also improve the semantics or quality of health information on the web.

\section{Conclusion}

The chapter highlights the potentials of IoT and Semantic web in healthcare sector. It shows that IoT and SWT have become significant medical tools with the capacity to improve health analytics and predictions, patient-physician communication, relationship and medical service and product delivery. With IoTpowered devices, report and response to critical health emergencies can be better managed. Also, medical data will be better stored and health workers can easily share relevant data needed to reduce errors and enhance effective treatment and other medical decisions. We conclude that IoT and SWT will play more prominent role in the future of healthcare if more investments are made to expand its availability, functionality and accessibility. However, apt security 
measures need to be implemented by stakeholders to enhance clinical data integrity and security. Thus, there is the need for authorities and hospitals to invest more in heath technologies such as IoT to enhance their capacities to respond to emerging health realities. In the future, we intend to examine the readiness of the health sector towards the adoption and implementation of smart health technologies like IoT-based devices in Nigeria.

\section{References}

Ali, D and Samina, R.A.(2009). Computerizing clinical pathways ontology-based modelling and execution, Medical informatics in a united and healthy Europe, Ios press. Uk.

Cheung, K.-H. ; Yip, K. Y; Smith, A; Deknikker, R. A; and Gerstein,M. (2005). ASemantic web use case for integrating data in the life sciences domain," Bioinformatics, 21 (1):85-96.

David, H.R. and Kyle, D.C. (2015). The Digital Revolution comes to US Healthcare. Goldman Sachs Global Investment Research, 1-54.

Available online via: https://www.wur.nl/upload_mm/0/f/3/8f e8684c-2a84-4965-9dce-

550584aae48c_Internet\%20of\%20Things \%205\%20\%20Digital\%20Revolution\%20 Comes\%20to\%20US\%20Healtcare.pdf Accessed 20 August, 2021.

Daniel, C and Cody V. K. B (2019). Semantic Enrichment of Streaming Healthcare Data. medRxiv preprint doi: https://doi.org/10.1101/2019.12.01.1901 $\underline{3615}$

Dimitrov, DV. (2016). Medical internet of things and big data in healthcare. Healthcare Informatics Research. 22 (3):156-163.

Erdogan, D. (2009). Semantic Web in eHealth. ACMSE '09 March 19-21, 2009, Clemson, SC, USA.
Eero , H; Kim,V and Osma, S. (2008). Health Finland Finnish Health Information on the Semantic Web", Semantic computing research group.

Gnanambal , S and Thangaraj, M (2010). Research Directions in Semantic Web on Healthcare. Int. J. of Comp. Scie. and Info. Techno, 1 (5): 449-453

Hristidis V. (2010). Information Discovery on Electronic Health Records. New York: CRC Press (taylor \& francis group).

Hossain M.S. \& Muhammad Ghulam (2016). Cloud-assisted Industrial Internet of Things (IIoT) - Enabled framework for health monitoring. Computer Networks. Available at:

http://dx.doi.org/10.1016/j.comnet.2016. $\underline{01.009}$.

Hadeel, K.H; Jameel, K.A; and Muthana A.W. (2021) The IoT for Healthcare Applications. IOP Conf. Ser.: Mater. Sci. Eng. 1105012075. doi:10.1088/1757-899X/1105/1/012075

https://www.iotnow.com/2021/02/26/108036-the-roleof-iot-in-healthcare-during-covid-19/

https://medium.com/datadriveninvestor/ 7-staggering-stats-on-healthcare-iotinnovation- fe6b92774a5c

https://www.reportsanddata.com/reportdetail/iot-in-healthcare-market

https://www.gsma.com/mobilefordevelop ment/blog/iot-applications-in-the-fightagainst-covid-19/

https://www.tutorialspoint.com/internet_ of_things/index.htm

Wipro website (2021). Business Process what can IoT do for Healthcare? Available at: https://www.wipro.com/businessprocess/what-can-iot-do-for-healthcare-/

Islam, S. M. R; D. Kwak, M. H. Kabir, M. Hossain, and K.-S. Kwak,(2015). Internet of Things for health care: a comprehensive survey," IEEE Access, 3: 678-708. 
Kang, L; L. Yi, and L. Dong (2014). "Research on construction methods of big data semantic model," in Proceedings of the World Congress on Engi, 1: 2-4

Lee, $\mathrm{F}$; Ivan $\mathrm{H}$; Tonya, $\mathrm{H}$; Eric, $\mathrm{N}$; and Susie $S$ (2007).The Semantic Web in Action. Scientific American INC. 91-97. Available at $\mathrm{w}$ w w. S c i A m . c o m. Accessed August, 2021.

Mohd, J and Ibrahim, H.K. (2021). Internet of Things (IoT) Enabled Healthcare Helps to take the Challenges of COVID-19 Pandemic. Journal of Oral Biology and Craniofacial Re. ,11, 209-214

Mahtab, K and Azin R. (2019). Semantic Web Technologies for Sharing Clinical Information in Health Care Systems. ACTA INFORM MED. 2019 MAR 27(1): 47. doi: 10.5455/aim.2019.27.4-7

Mohammed, D and Ahmed, M (2019). IOT Service Utilization in Healthcare, IntechOpen. Available at www.intechopen.com. DOI: http://dx.doi.org/10.5772/intechopen.860 14

Mahadevaiah, G. (2020). Machine learning \& semantic web technologies for cancer care. Maastricht University. https://doi.org/10.26481/dis.20200409g $\mathrm{m}$

Onyema, E.M; A. Sharma., C.E, Nwafor., A.G. Fyneface., S, Sen., E.C. Edeh (2020a). Impact of Emerging Technologies on the Job Performance of Educators in Selected Tertiary Institutions in Nigeria. The Journal of Computer Scie. and its Application, 27 (1), 52-62. https://dx.doi.org/10.4314/jcsia.v27i1.4

Onyema, E.M; Nwafor, C.E; Faith, A.O; Shuvro, S; Fyneface, G.A; Aabha, S; A.O. (2020b). Impact of Coronavirus Pandemic on Education. Journal of Education and Practice, 11 (13), 108-121.

Onyema, E.M. (2019). Opportunities and Challenges of use ofwear Mobile Phone Technology in teaching and learning in Nigeria- A Review. International Journal of Research in Engineering and Innovation, 3(6), 352-358. http://doi.org/10.36037/IJREI.2019.3601
Onyema, EM; PK Shukla, S Dalal, M Mathur, M Zakariah, B Tiwari (2021). Enhancement of Patient Facial Recognition through Deep Learning Algorithm: ConvNet. Hindawi Journal of Healthcare and Engineering, 38, 154-166.

Partha P.Ray (2014). Home Health Hub Internet of Things (H3IoT): An architectural framework for monitoring health of elderly people. IEEE32331 International Conference on Science, Engineering and Management Research (ICSEMR 2014) pp.3-5. Available at:

http://ieeexplore.ieee.org/document/704 3542/\%0

Ahttp://ieeexplore.ieee.org/xpls/abs_all.js p?arnumber $=7043542$.

Patel, K.K.; Patel, S.M (2016). Internet of things-IoT: definition, characteristics, architecture, enabling technologies, application \& future challenges. Int. J. Eng. Sci. Comput., 6: 6122- 6131.

Pathak, J, R. Kiefer, C. Chute (2012). Using Semantic Web Technologies for Cohort Identification from Electronic Health Records for Clinical Research". AMIA Jt Summits Transl Sci Proc: 10-19

Rafat H, Malek B , Bilal H. A. (2020). A Semantic-Based Approach for Managing Healthcare Big Data: A Survey, Hindawi Journal of Healthcare Engin, 1-12. https://doi.org/10.1155/2020/8865808

Shah, N; Yasir, A.U and Ivan G. (2019). Internet of Things for Healthcare Using Effects of Mobile Computing: A Systematic Literature Review. Hindawi Wireless Comm. and Mobile Computing. 1-https://doi.org/10.1155/2019/5931315

Shariq, A.B; Muhammad, W.A; Syed, G and Onyema, EM. (2021). Smart Health Application for Remote Tracking of Ambulatory Patients. In SK Hafizul Islam and Debabrata Samanta Ed; Smart Healthcare System design: Security and Privacy Aspects. Chapter 2 Page 33-55. https://doi.org/10.1002/9781119792253. ch2 
Tiwari,S.M; Sarika, J, Ajith, A and Smita, S. (2019). Secure Semantic Smart HealthCare (S3HC). Journal of Web Engineering, 17 (8), 617-

646 doi: https://doi.org/10.13052/jwe15 40-9589.1782

Umair, M.; Cheema, M.A.;Cheema, O.; Li, H.; Lu, H. (2021). Impact of COVID-19 on IoT Adoption in Healthcare, Smart Homes, Smart Buildings, Smart Cities, Transportation and Industrial IoT. MDPI Sensors, 21: 3838.

https://doi.org/10.3390/s21113838

Verma P., Sood S.K. and Kalra S. (2017). Cloud-centric IoT based student healthcare monitoring framework.
Journal of Ambient Intelligence and Humanized Computing, 0(0), pp.1-17. Available at: http://dx.doi.org/10.1007/s12652-0170520-6.

Vermesan, O. and Friess, P. (2014). Internet of Things-From Research and Innovation to Market Deployment. In: River Publishers. US.

Yap, S.Y and Tan, C.C. (2020). tudy of Internet of Things Enabled Healthcare Acceptance in Malaysia, Journal of critical reviews. 7 (3), 25-32. 